\title{
Towards Optimal h-p Adaptation Near Singularities in Finite Element Electromagnetics
}

\author{
Dennis Giannacopoulos and Steve McFee \\ Computational Analysis and Design Laboratory \\ Electrical Engineering Department, McGill University \\ 3480 University Street, Montreal, PQ, H3A 2A7, Canada
}

\begin{abstract}
One of the most important problems of hybrid $h$ - $p$ adaption in finite element electromagnetics has been the accurate and efficient resolution of the singularities associated with sharp material edges and corners. One of the key obstacles has been the lack of objective standards by which to evaluate and compare adaptive control strategies. A set of optimal adaption benchmarks for the fundamental electromagnetic point and line singularity models is presented. The primary adaption procedures and control schemes are evaluated and compared. The absolute and relative performance of the competing approaches is discussed.
\end{abstract}

\section{INTRODUCTION}

Currently, finite element analysis (FEA) is widely used in electromagnetic design - typically, FEA tools are used to computationally simulate and evaluate the performance of a new device design before building a prototype. Today, the state-of-the-art in FEA research lies in the development of adaptive solver technologies. In the future, it is believed that adaptive solvers will be able to reliably compute the performance of a proposed device to within the engineer's specified tolerances.

Today, three basic adaption models are under study: $h$ type; - -type; and combined $h$ - and $p$-type (called $h$-p). Essentially, these models only differ in the techniques used to update the finite element discretization within the adaptive feedback loop (described below).

A. Generate initial discretization.

Repeat:

B. Solve finite element problem.

C. Evaluate solution accuracy; if adequate STOP.

D. Identify regions of inadequate discretization.

E. Update finite element discretization. Until STOP.

Simply stated, $h$-adaption adds elements to the mesh to improve a discretization; $p$-adaption increases element orders within the mesh to improve a discretization; and $h-p$ adaption employs a combination of both procedures.

While $h$-adaption has become increasingly popular in electromagnetic FEA research during the past ten years [1][4], and more recently, effective $p$-adaption codes have started to emerge [5], [6], practical $h-p$ adaptive strategies for electromagnetic FEA still remain out of reach. One important reason for this slow progress - aside from the inherent complexity of implementing and controlling $h-p$ adaption - is the lack of objective benchmarks by which to measure the merits and flaws of adaptive strategies.

One of the most important challenges for all types of adaption in FEA is the accurate and efficient resolution of the singularities associated with sharp material edges and corners [7]. The purpose of this contribution is to present a set of adaption benchmarks for these singularities, and illustrate their usefulness in the analysis and design of optimal $h-p$ adaption strategies.

\section{ADAPTION BENCHMARKS}

The adaption analyses are based on the fundamental point-charge and line-current singularity models. The following benchmarks were computed for each model.

1. Optimal $h$-adaption (Orders 1 and 2).

a) All nodes free to move with each adaptive step.

b) Only new nodes free to move in an adaptive step.

c) All new nodes set by element bisection.

2. Optimal p-adaption (Orders $1,2,4$, and 8).

a) All element orders updated uniformly with each adaptive step.

b) Element orders updated non-uniformly (mixed-order p-adaption) in an adaptive step.

3. Optimal $h-p$ adaption (Orders $1,2,4$, and 8).

a) Decoupled: $h$-followed by $p$-adaption.

b) Fully integrated $h$-and $p$-adaption.

In this work, optimal adaption implies optimal functional convergence. The different types of adaption techniques considered here are intended to represent a range of methods varying in computational cost and practicality of implementation. For each case, new degrees of freedom (DOF) are added to the discretization so as to yield the best possible improvement in functional value for each adaptive step. The optimal placement of the new nodes is determined by solving a full-domain constrained optimization problem at each adaptive step [8]. 


\section{RESULTS}

A set of 50 optimal adaption benchmarks were computed for the two singularity models. Each benchmark is based on the resolution of a region close to - but excluding - the singularity under consideration. A radial neighbourhood spanning a 100-fold decay in potential was used. In order to obtain the best possible resolution in the rate of convergence for the optimal adaption methods studied in this work, each adaptive iteration was based on increasing the number of (DOF) in a discretization by the minimal increments appropriate to the type of adaption being considered. The following results represent a crosssection of the benchmarks computed.

\section{A. Optimal Meshes}

Fig. 1 illustrates the radial discretization, for the point singularity model, of a range of $h$-adaption optimized meshes in which all nodes were free to move in each adaptive step. Both first- and second-order meshes are presented, but only the geometric nodes are plotted. It is noted that for the same number of DOF, in the point singularity model, the resulting distributions of DOF will differ between the first- and second-order meshes when all nodes are free to move in each adaptive step. The corresponding results for the line singularity model have the interesting property that the first- and second-order optimal $h$-adaption meshes have exactly the same distribution of DOF when all nodes are free to move with each adaptive step. The radial discretizations are plotted on a logarithmic scale because of the proximity of some of the nodes to each other near the singularity. Fig. 2 shows a range of the different types of optimal $h$-adaption secondorder meshes for the line singularity model: $\mathrm{A} \rightarrow$ all nodes free to move; $\mathrm{B} \rightarrow$ only new nodes free to move; $\mathrm{C} \rightarrow$ new nodes set by element bisection. It is interesting to note the

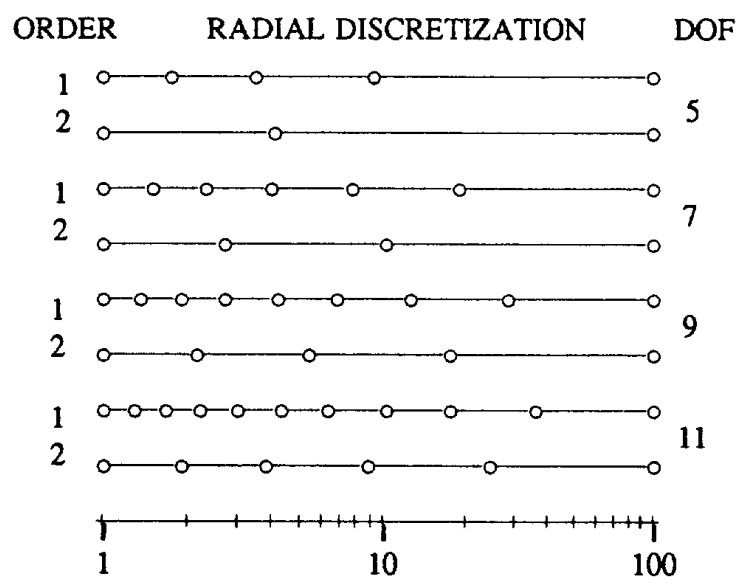

Fig. 1. Optimal $h$-adaption radial discretizations with all nodes free to move in each adaptive step for the point singularity model.

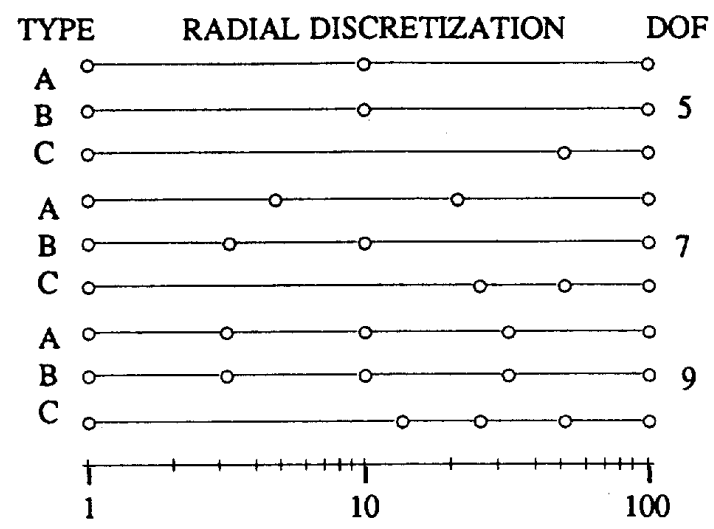

Fig. 2. Optimal $h$-adaption meshes for the line singularity model using second-order elements.

first two types of optimal $h$-adaption represented in Fig. 2 result in meshes with highly refined discretizations near the singularity, while the optimal meshes obtained with element bisection using second-order elements, can not resolve the singularity as rapidly. Similar results were obtained for the point singularity. The meshes obtained for both models, when using the first two types of optimal $h$-adaption, have very similar distributions of DOF. This may suggest some practical techniques for obtaining 'near' optimal $h$-adaption meshes at a cost less than would be required for the best possible case when all nodes are free to move in each adaptive step.

Fig. 3 compares evolving optimal $h-p$ adaptive discretizations for the point singularity: $\mathrm{A} \rightarrow$ integrated $h-p$ adaption; $\mathrm{B} \rightarrow$ decoupled $h-p$ adaption. In each adaptive step, the integrated $h-p$ adaptive solver improves the discretization by either bisecting an element or increasing

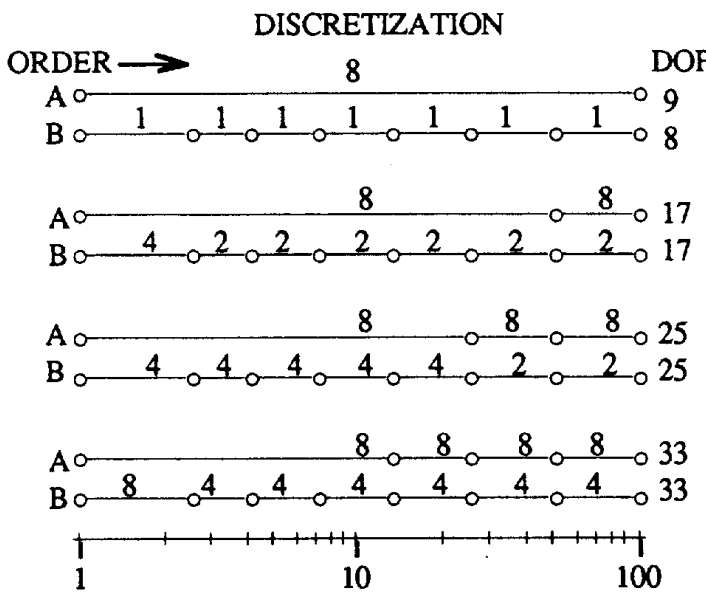

Fig. 3. Evolving discretizations for optimal h-p adaption for the point singularity. 


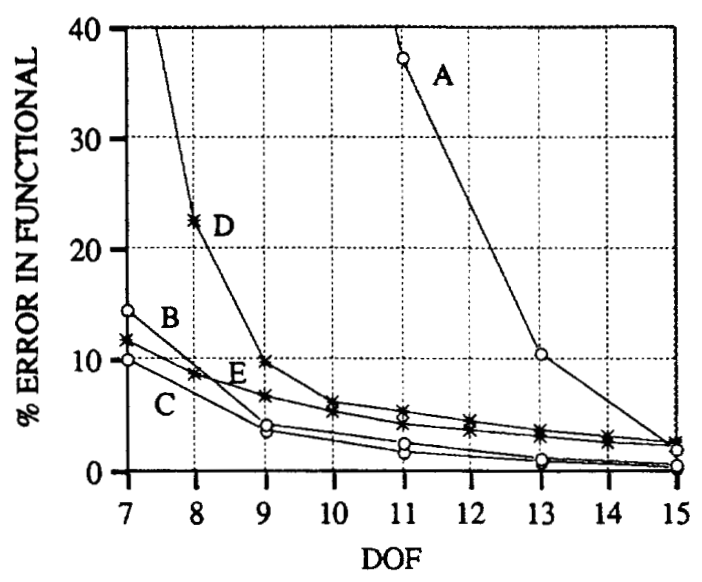

Fig. 4. Optimal $h$-adaption convergence (point singularity).

the order of an element, such that the optimal improvement in the functional value is achieved. The decoupled $h-p$ adaptive solver considered here, first refines the mesh by an optimal element bisection algorithm until a given rate of convergence is attained, and then improves the discretization by increasing the order of an element in each subsequent adaptive step (i.e., mixed-order $p$-adaption). The two types of $h-p$ adaption corresponding to Fig. 3, result in rather different types of optimal discretizations. The integrated approach, initially, attempts to resolve the singularity by increasing element order rather than by element bisection. The decoupled approach, considered in this instance, seems to result in a better distribution of DOF since it produces optimal meshes with a higher density of DOF near the singularity, compared with the more uniform distribution produced by the integrated approach. Analogous results were obtained for the line singularity.

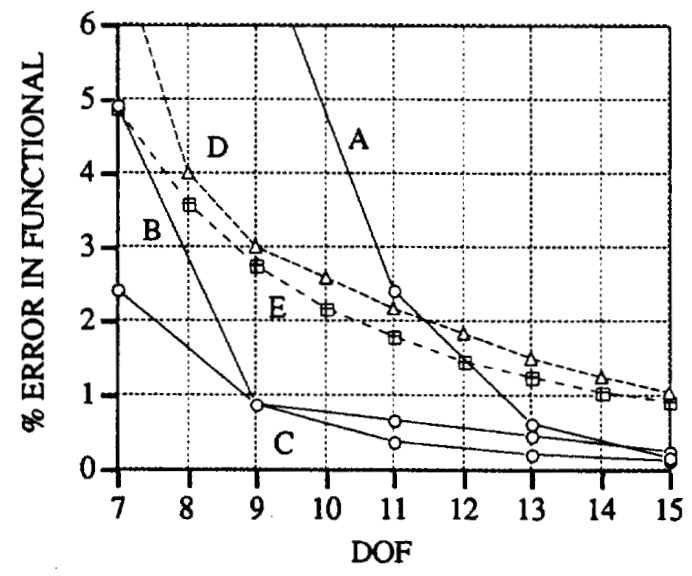

Fig. 5. Optimal $h$-adaption convergence (line singularity).

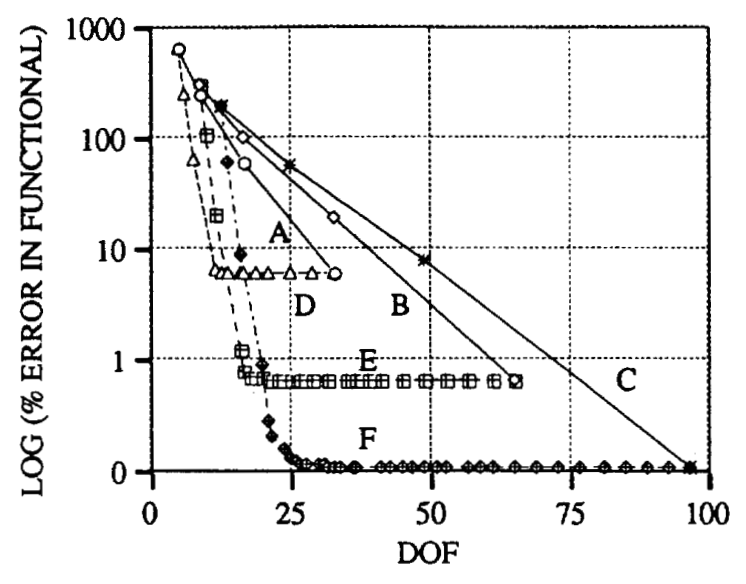

Fig. 6. Optimal p-adaption convergence (point singularity).

\section{B. Optimal h-adaption Convergence}

Fig. 4 and Fig. 5 illustrate the optimal convergence of a range of $h$-adaption strategies applied to the point and line singularity models, respectively: $\mathrm{A} \rightarrow$ element bisection ( $2^{\mathrm{ND}}$ order elements); $\mathrm{B} \rightarrow$ new nodes free to move in each step ( $2^{\mathrm{ND}}$ order elements); $\mathrm{C} \rightarrow$ all nodes free to move in each step ( ${ }^{\mathrm{ND}}$ order elements); $\mathrm{D} \rightarrow$ element bisection ( $1^{\text {ST }}$ order elements); $\mathrm{E} \rightarrow$ all nodes free to move in each step ( $1^{\text {ST }}$ order elements). The two singularity models display similar characteristics in terms of their optimal $h$ adaption convergence. For second order elements, the strategy in which only new nodes are free to move in each adaptive step has comparable error convergence with that of the computationally more expensive strategy in which all nodes are free to move in each adaptive step. The optimal element bisection for second-order elements, by contrast, converges comparatively slowly. When first-order elements

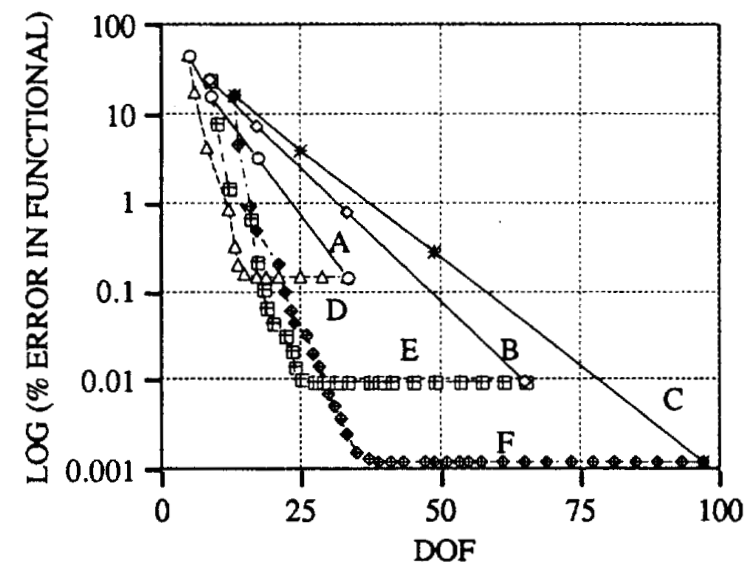

Fig. 7. Optimal p-adaption convergence (line singularity). 


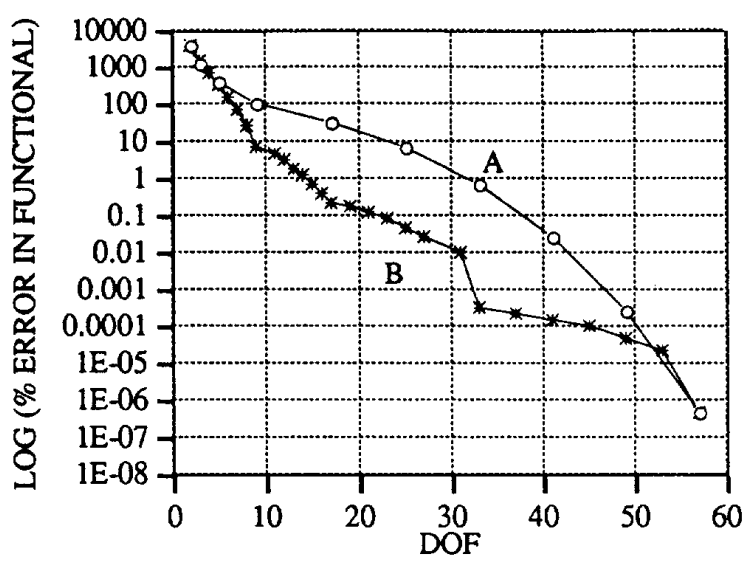

Fig. 8. Optimal $h-p$ adaption convergence (point singularity).

are employed, however, the relative performance of the bisection strategy, with respect to the fully optimal (all nodes free to move) first-order strategy, improves. This is due primarily to the more rapid resolution of the singularity that is possible with a first-order element bisection scheme than with its second-order counterpart. That is, for the same number of DOF, a first-order bisection strategy can distribute the DOF more effectively than a similar second-order approach, at least until a certain error tolerance level $(\sim 2 \%)$.

\section{Optimal p-adaption Convergence}

Fig. 6 and Fig. 7 illustrate the optimal convergence of p-adaption strategies applied to a range of uniform initial meshes for the point and line singularity models, respectively: $\mathrm{A} \rightarrow 4$ elements, uniform $p$-adaption; $\mathrm{B} \rightarrow 8$ elements, uniform $p$-adaption; $C \rightarrow 12$ elements, uniform $p$-adaption; $\mathrm{D} \rightarrow 4$ elements, mixed-order $p$-adaption; $\mathrm{E} \rightarrow$ 8 elements, mixed-order $p$-adaption; $F \rightarrow 12$ elements, mixed-order $p$-adaption. For the two singularity models, when starting from uniform initial meshes, mixed-order $p$ adaption results in a faster rate of convergence. When starting from $h$-adaption optimal meshes obtained with all nodes free to move, however, it was observed that the difference in the rates of convergence between uniform- and mixed-order $p$-adaption is not as substantial as that shown in Fig. 6 and Fig. 7. Also, it has been observed in previous work that uniform order $p$-adaption becomes more efficient than optimal $h$-adaption, once the mesh is sufficiently well refined using an optimal $h$-adaption strategy.

\section{Optimal h-p adaption Convergence}

Fig. 8 illustrates the optimal convergence for two $h-p$ adaption strategies for the point singularity, specifically, those considered previously in Fig. 3: $\mathrm{A} \rightarrow$ integrated $h-p$ adaption; B $\rightarrow$ decoupled $h-p$ adaption (optimal element bisection followed by mixed-order $p$-adaption). Evidently, the decoupled approach is able to distribute the DOF more efficiently, and therefore, provides a faster rate of convergence. Similar results were obtained for the line singularity model.

\section{CONCLUSIONS}

A set of important optimal adaption benchmarks has been presented. Based on a comparison of these results it is clear that a very rapid variation in discretization is required to resolve singularities efficiently. Therefore, adaption strategies which can best combine the advantages of efficient distribution of DOF of both $h$ - and p-type adaption, will provide the most desirable convergence rates. Theoretically, decoupled $h-p$ adaption can never produce better convergence performance than fully integrated $h-p$ adaption. However, based on the results presented in this contribution, the most effective practical implementation of $h-p$ adaption is a decoupled approach. For example, such an approach might use an optimal $h$-adaption method based on low-order elements to refine the mesh initially, until a suitable convergence criterion is satisfied, and then employ an optimal p-adaption strategy to meet a more stringent criterion.

There are several types of error measures which may be used in adaptive FEA [9], [10]. For this work, however, adaptive refinement was based on optimal functional values. The principal reason for this choice is associated with the mathematical formulation used, which is based on variational finite element methods. Future investigations into the characteristics of optimal $h-p$ adaption near singularities in finite element electromagnetics, might include a comparison of various types of error measures.

\section{REFERENCES}

[1] P. Femandes, P. Girdinio, P. Molfino, G. Molinari and M. Repeno, IEEE Trans. Magn. Vol. 26(2), pp. 795-798, 1990.

[2] J. Penman and M.D. Grieve, IEEE Trans. Ind. Appl, Vol. 24(6), pp. 1042-1049, 1988.

[3] Z.J. Cendes and D.N. Shenton, IEEE Trans. Magn. Vol. 21(5), pp 1811-1816, 1985.

[4] T. Tamhuvud, K. Reichert and J. Skoczylas, IEEE Trans. Mapn. Vol. 26(2), pp. 779-782, 1990.

[5] S. McFee and J.P. Webb, IEEE Trans. Magn., Vol. 28(2), pp. 1708$1711,1992$.

[6] A. Raizer, S.R.H. Hoole, G. Meunier and J.-L. Coulomb, Jour. Appl. Phys. Vol. 67(9), pp. 5803-5805, 1990.

[7] W. Rachowicz, J.T. Oden and L. Demkowicz, Comp. Meth. Appl. Mech. Vol. 77, pp. 181-212. (Also: Parts 1 \& 2, pp. 79-180).

[8] J.E. Dennis Jr., and R.B. Schnabel, Numerical Methods for Unconstrained Optimization and Nonlinear Equations, Prentice-Hall, Englewood Cliffs, NJ, 1983.

[9] N. A. Golia s and T.D. Tsiboukis, IEEE Trans. Magn., Vol. 29(2), pp. 1886-1889, 1993.

[10] G. Drago, P. Molfino, M. Nervi, and M. Repetto, IEEE Trans. Magn, Vol. 28(2), pp. 1743-1746, 1992. 